\title{
ENERGY LOCALIZATION THROUGH BRAGG GRATINGS IN QUADRATIC MEDIA FOR SECOND HARMONIC GENERATION
}

\author{
C. Conti, G. Assanto* \\ Department of Electronic Engineering, Terza University of Rome \\ Via della Vasca Navale 84, 00146 Rome, Italy \\ AND S. TRILLo \\ Fondazione Ugo Bordoni \\ Via Baldassarre Castiglione 59, 00142 Rome, Italy
}

In a waveguide for second-harmonic generation, a linear corrugation able to couple counterpropagating waves at the second-harmonic and/or at the fundamental frequency can induce localization effects through the formation of gap-simultons, i.e. bi-color gap-solitòns. These can move slowly or be stationary, collide and merge. All-optical memories are envisaged.

PACS numbers: $42.65 .-\mathrm{k}, 42.65 . \mathrm{Pc}, 42.65 . \mathrm{Ky}$

A nonlinear optical response coupled with feedback mechanisms is able to support optical multistability in various material systems and geometries. In periodically perturbed waveguides such as distributed feedback gratings (DFBG), a Bragg resonance coupling counterpropagating fields can associate with a Kerr or a parametric nonlinearity to give rise to transmissive nonlinear eigenstates within the otherwise propagation-forbidden photonic bandgap [1-5]. These correspond to energy localization states in the form of stationary or slowly travelling "gap-solitons": when properly excited, they can be viewed as optical bits trapped within the DFBG for short- or long-term storage depending on their propagation speed. Due to the rapidly growing interest in transparent optical networks and all-optical memories, the possibility of stationary localized states or "still" gap-solitons in DFBGs is an intriguing one.

In this paper, after introducing two-color quadratic gap-solitons or gap-simultons in a channel waveguide for frequency doubling, we address the problem of excitation of slowly moving or stationary states in singly (srDFBG) or doubly resonant gratings (drDFBG), showing that the feasibility of read/write all-optical memories is within experimental reach. In a material system with a quadratically

\footnotetext{
*e-mail: Assanto@ele.uniroma3.it
} 
nonlinear response, restricting - with no lack of generality - to the case of quasi-plane waves and SHG in a waveguide supporting fundamental and second harmonic (SH) modes of wave vectors $\beta_{1}$ and $\beta_{2}$, respectively, in the presence of a shallow corrugation of period $\Lambda \cong \pi / \beta_{1}$ (Bragg resonance at the fundamental frequency $\omega$ ), the overall response can be described by the four equations [3]:

$$
\begin{aligned}
& \phi_{1 \tau}^{ \pm}=\mp \phi_{1 \xi}^{ \pm}+\mathrm{i} \Delta_{1} \phi_{1}^{ \pm}+i \phi_{1}^{\mp}+i\left(\phi_{1}^{ \pm}\right)^{*} \phi_{2}^{ \pm}, \\
& \phi_{2} \tau^{ \pm}=\mp v \phi_{2 \xi}^{ \pm}+\mathrm{i} v \Delta_{2} \phi_{2}^{ \pm}+\mathrm{i} v \kappa \phi_{2}^{\mp}+\mathrm{i} v\left(\phi_{1}^{ \pm}\right)^{2} / 2,
\end{aligned}
$$

where ${ }^{+}$and - refer to forward and backward propagating waves, respectively; subscripts 1 and 2 label fundamental frequencies (FF) and $\mathrm{SH}$ components; $v=v_{g 2} / v_{g 1}$ and $\kappa=\kappa_{2} / \kappa_{1}$ are the ratios of group velocities and Bragg coupling strengths at $2 \omega$ and $\omega$, respectively, $\Delta_{1,2}=\Delta \beta_{1,2} /\left(2 \kappa_{1}\right)$ are normalized detunings with $\Delta \beta_{m}=2 \beta_{m}-2 \pi m / \Lambda(m=1,2), \tau=\kappa_{1} v_{g 1} t$ and $\xi=\kappa_{1} z$ are normalized temporal and propagation coordinates, respectively. Notice that the case $\kappa=0$ corresponds to a grating with no bearing on the SH fields, i.e. a singly resonant structure (srDFBG). Starting from the linear Bloch eigenfunctions, through a multiple scales perturbative method, assuming $\kappa>0$ and both frequencies close to their lower (in-gap) bandedges, system (1) reduces to [3]

$$
\begin{aligned}
\phi_{1 \tau} & =-\frac{\mathrm{i}}{2} \phi_{1 \xi \xi}+\frac{\mathrm{i}}{\sqrt{2}} \phi_{1}^{*} \phi_{2} \exp (-\mathrm{i} \beta \tau), \\
\phi_{2 \tau} & =-\frac{\mathrm{i} v}{2 \kappa} \phi_{2 \xi \xi}+\frac{\mathrm{i} v}{2 \sqrt{2}} \phi_{1}^{2} \exp (\mathrm{i} \beta \tau),
\end{aligned}
$$

with $\phi_{1}$ and $\phi_{2}$ the envelopes at $\omega$ and $2 \omega$, respectively, and $\beta=2\left(\Delta_{1}+1\right)$ $-v \kappa\left(1+\Delta_{2} / \kappa\right)$ playing the role of a phase mismatch. System (2) looks like the standard set of equations describing SHG interaction in bulk, but with the role of time and space interchanged. Such set is known to admit stable soliton-like solutions of the bright-bright kind which, in the present context, are stationary or slowly-moving gap-simultons within the DFBG [6, 7]. These localized states or simultons are the sought outcome of a balance between grating dispersion and nonlinearity [8]. Their excitation, however, can only be investigated with the aid of system (1) via numerical propagation through the boundaries (homogeneous-linear/ periodic-nonlinear) of the structure. Upon launching a fundamental frequency pulse of Gaussian shape $\phi_{1}^{+}(0, \tau)=A_{1} \exp \left[-\left(\tau / \tau_{0}\right)^{2}\right]$ at $\xi=0$ in a singly or doubly resonant DFBG, a portion of the incident FF light is reflected together with the SH generated at the interface and inside the grating, whereas coupled FF and SH components can parametrically interact and propagate forward as a simulton, as shown in Fig. 1 for a srDFBG and in Fig. 2 for a drDFBG. The SH field which in Fig. 1 appears to depart from the simulton is the free $\mathrm{SH}$ wave, which is not present in a drDFBG (Fig. 2) because of the SH Bragg coupling. Conversely, $\mathrm{FF}$ and $\mathrm{SH}$ interacting components propagate at a group velocity close to $0.3 v_{g 1}$. This behaviour, indeed, is a typical one: the excitation of either an srDFBG or a drDFBG, provided the conditions for existence are satisfied (i.e., high input intensity at $\omega$ and $\Delta_{1} \Delta_{2}<0$ for srDFBGs, or $\Delta_{1} \approx-1$ and $\Delta_{2} \geq-k$ for drDFBGs), gives origin to a slowly-travelling gap simulton [9]. 


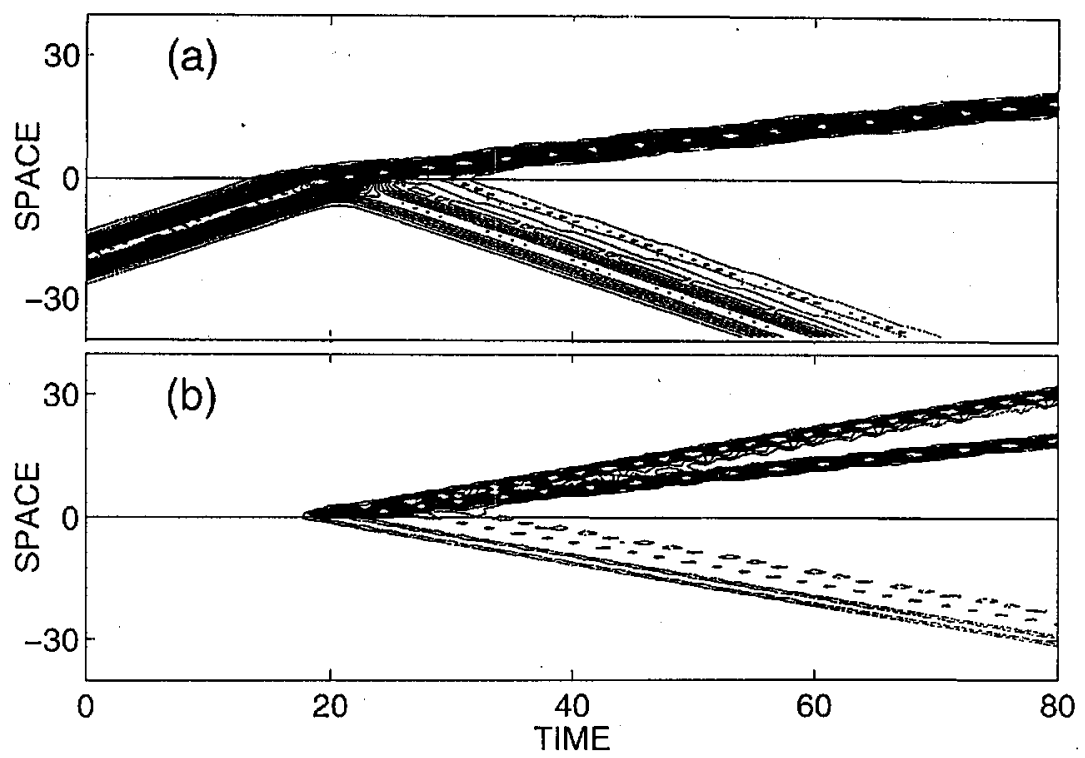

Fig. 1. Excitation of a two-color gap soliton in a singly resonant grating. The contour levels show total FF (a) and SH (b) intensities versus space $\xi$ and time $\tau$ from Eqs. (1) with $v=0.5, \kappa=0, \Delta_{1}=-0.9$, and $\Delta_{2}=5$.

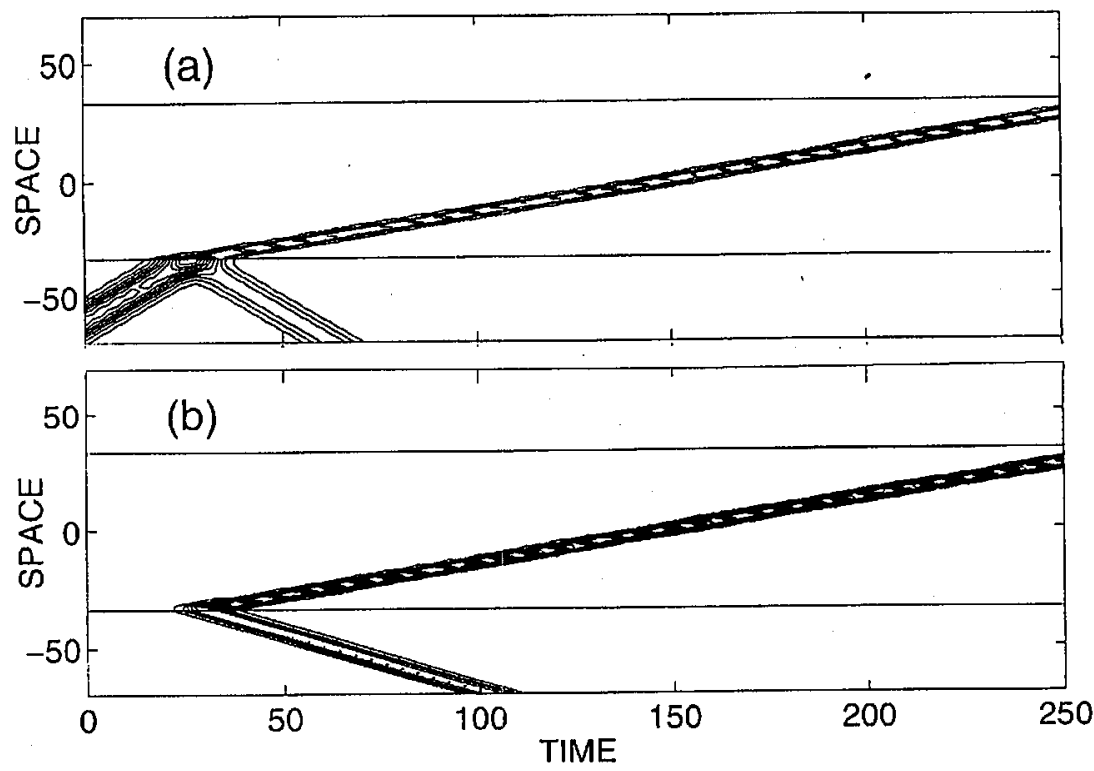

Fig. 2. Excitation of a two-color gap soliton in a doubly resonant grating. The contour levels show total FF (a) and SH (b) intensities versus space $\xi$ and time $\tau$ from Eqs. (1) with $v=0.5, \kappa=1, \Delta_{1}=-0.9$, and $\Delta_{2}=-0.9$. 
The formation of a "still" simulton, however, based on intuitive considerations on momentum conservation, appears nontrivial. With such constraint in mind, an approach to forming a zero-momentum simulton is to resort to the inelastic collision of two counterpropagating but otherwise similar gap simultons in a drDFBG. This process is expected to conserve total momentum and give rise - through merging - to a zero group velocity state in the grating. Such possibility, inspired by the non-integrable nature of the governing equations and by numerical results obtained with reference to in-phase quadratic spatial solitons interacting with opposite transverse velocities [10], can be explored by launching identical beams from opposite ends of the Bragg structure. Figure 3 shows that the expected behaviour is indeed verified: two in-phase and counterpropagating FF inputs in the grating evolve into slow simultons which, after collision, merge in a single two-color localized state. Notice that this process, for parameters close to those in the example, allows one to reach a final bound state carrying about $70 \%$ of the energy associated with the incoming gap-simultons, despite radiation of energy occurring at both FF and SH [11]. In real units, a pulse duration of $100 \mathrm{ps}$ is estimated for an input peak intensity of $100 \mathrm{MW} / \mathrm{cm}^{2}$ into a nonlinear crystal with $d_{\mathrm{eff}}=12 \mathrm{pm} / \mathrm{V}\left(\right.$ e.g. $\left.\mathrm{KNbO}_{3}\right), \kappa 1=\kappa_{2}=0.5 \mathrm{~mm}^{-1}$ and $v_{g 1}=c / 2$. Figure 4 shows the collision phenomenology for two out-of-phase incoming.simultons: the merging is inefficient if they are in opposition, but holds to some extent even when they are in quadrature. The robustness of the coalescence effect for relative phases smaller than $\pi / 2$ is a rather encouraging characteristic of the "writing" process of a bit into this novel all-optical memory.

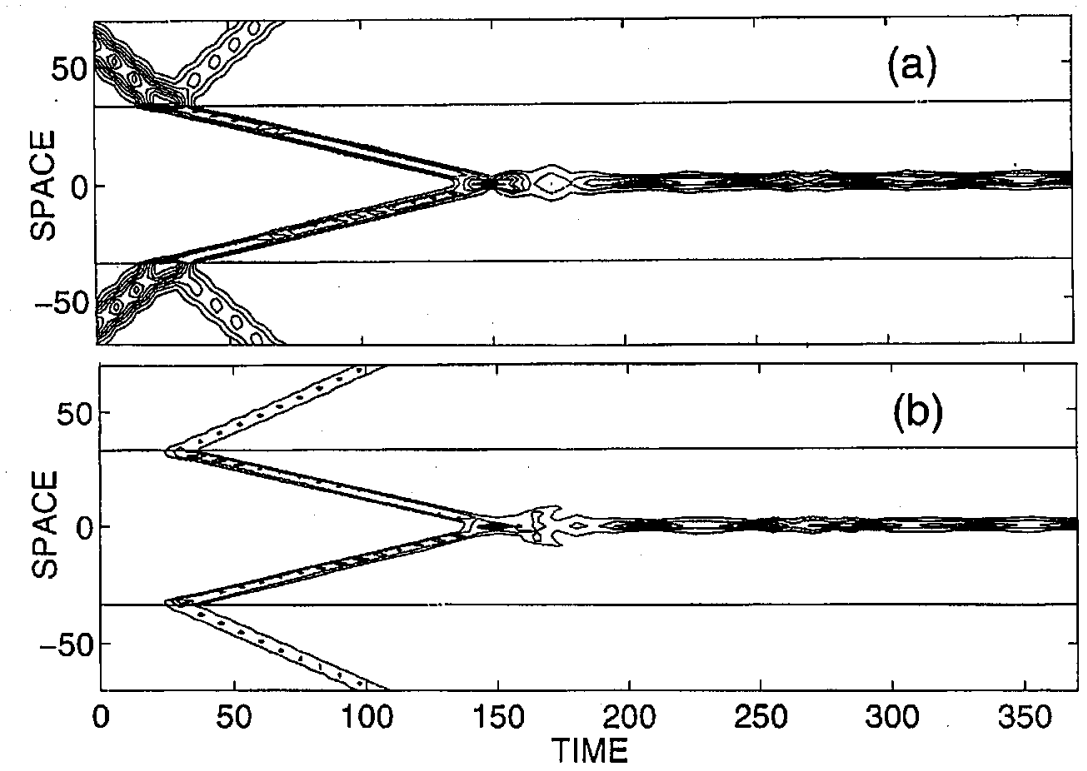

Fig. 3. Formation of a stationary gap soliton via the inelastic collision of two in-phase counterpropagating low-speed solitons in a doubly resonant grating: (a) FF; (b) generated SH (here $v=0.5, \kappa=1, \Delta_{1}=-0.9$, and $\Delta_{2}=-0.9$ ). 


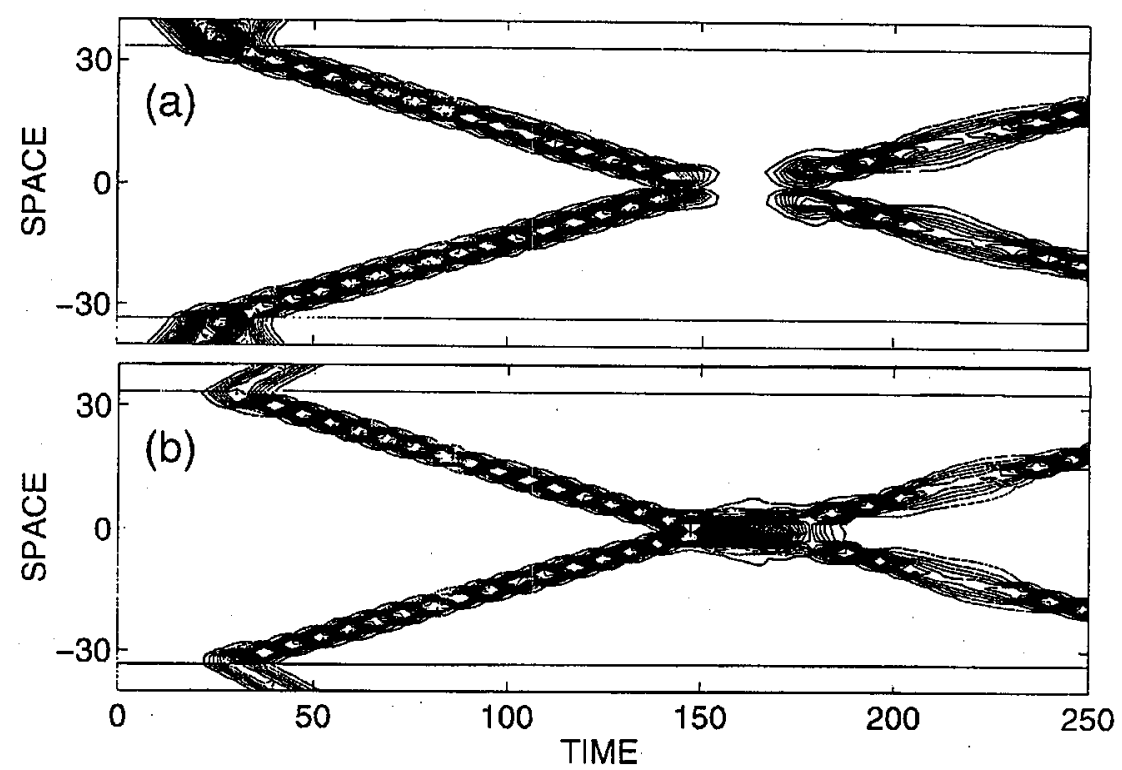

Fig. 4. Collision of two out-of-phase counterpropagating low-speed solitons in a doubly . resonant grating: (a) FF; (b) generated $\mathrm{SH}$ (here $v=0.5, \kappa=1, \Delta_{1}=-0.9$, and $\left.\Delta_{2}=-0.9\right)$.

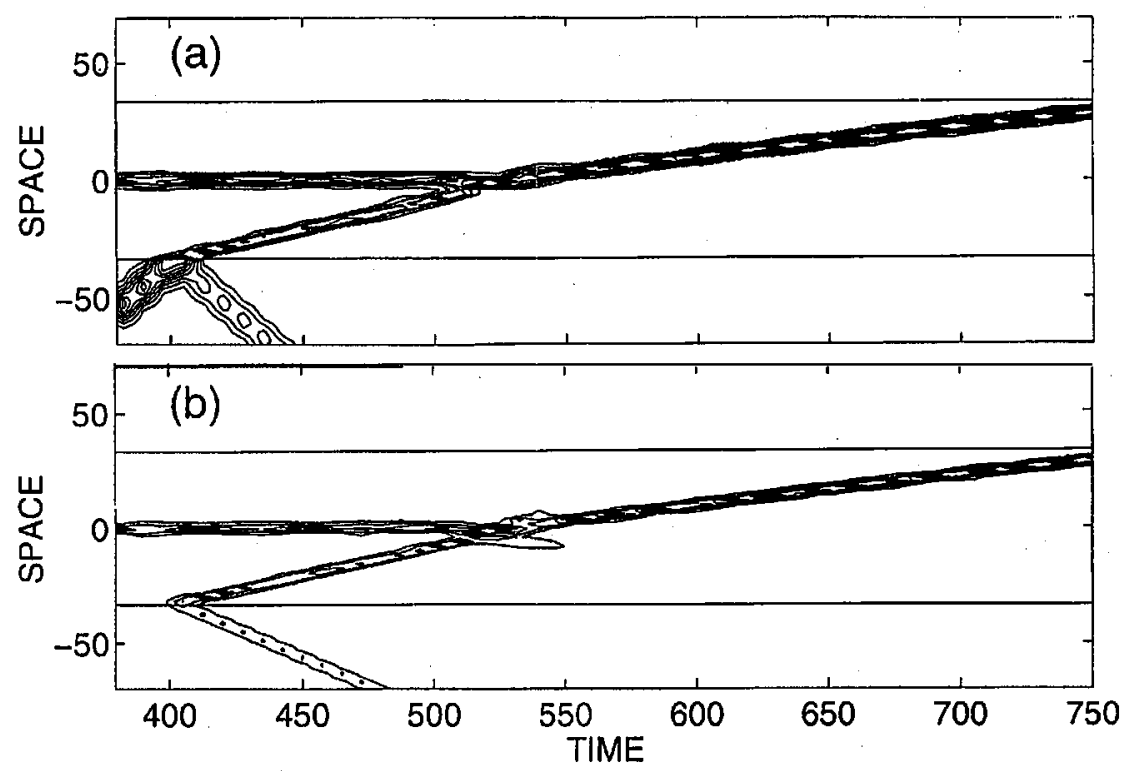

Fig. 5. Interrogation process of the still gap simulton formed in Fig. 3 by launching another slowly-moving soliton. (a) FF and (b) SH components. 
Since any (classical) detection process requires an interaction, an approach to revealing the presence of a still simulton, i.e. an optical bit in the DFBG-buffer, is the interrogation of the structure by means of another (similar) slowly travelling soliton, as sketched in Fig. 5. For an interrogating bit nearly in phase with the others, still (i.e.: stored bit) and moving solitons will coalesce once again, giving rise - this time - to an even slower state (because of a larger resulting "mass") which will eventually leave the drDFBG through the opposite end. The "reading" process does, therefore, "erase" the optical buffer, the state of which corresponds to the time of flight of the "inquiry" bit or beam [12]. Notice that this is a manifestation of the inherent bistability of a nonlinear photonics crystal, because the response depends on the "history" of the structure.

Quite different is, in the above context, the excitation of stationary simultons in srDFBGs. The governing equations (with $\kappa=0$ ), in fact, no longer resemble those ruling the propagation of quadratic spatial solitary waves. It is convenient to rewrite (1) including the frequency shift $\nu$ upon a gap-simulton moving at velocity $V$. This gives

$$
\begin{aligned}
& \mathrm{i}( \pm 1-V) u_{1 \zeta}^{ \pm}+\delta_{1} u_{1}^{ \pm}+u_{1}^{\mp}+\left(u_{1}^{ \pm}\right)^{*} u_{2}^{ \pm}=0 \\
& \mathrm{i}\left( \pm 1-V / v_{g 2}\right) u_{2 \zeta}^{ \pm}+\delta_{2} u_{2}^{ \pm}+\left(u_{1}^{ \pm}\right)^{2} / 2=0
\end{aligned}
$$

with $\zeta=\xi-V \tau, \phi_{m}^{ \pm}(\xi, \tau)=u_{m}^{ \pm}(\xi) \exp (\mathrm{i} m \nu \tau)(m=1,2), \delta_{1}=\Delta_{1}-\nu$ and $\delta_{2}=\Delta_{2}-2 \nu / v_{g 2}$. For a given detuning $\delta_{1}$, system (3) can be solved for any $\delta_{2}$ looking for bright-bright simulton-like solutions moving at velocity $V$, provided the condition of existence $\delta_{1} \delta_{2}<0$ is fulfilled (notice that, being $\nu$ typically small compared to $\Delta_{1,2}$, the latter translates into $\Delta_{1} \Delta_{2}<0$ for system (1)). To this extent, we can start from perturbative Thirring-like solutions valid for large $\delta_{2}[1,9]$, progressively reducing the latter parameter with a standard continuation technique. We are able to conclude that, as the phase mismatch (proportional to $\delta_{2}$ ) tends to vanishingly small values, the maximum simulton velocity approaches zero. The latter result can be restated as follows: bright-bright gap-simultons in a srDFBG exhibit lower and lower propagation velocities as the SH component (phase mismatch) becomes larger (smaller). Notice that larger fundamental peak powers lead to a smaller frequency shift $\nu$ and to a larger ratio between the simulton intensities at $\omega$ and $2 \omega$, respectively, therefore better approximating the Kerr phenomenology. Using a split-step algorithin we integrated system (3). Figure $6 a, b$ displays contour plots obtained at $\omega$ and $2 \omega$ versus propagation and time inside a srDFBG with $\Delta_{1}=-0.7$ and $\Delta_{2}=2$ : the fundamental input is partly reflected and partly transmitted upon generation of an $\mathrm{SH}$ component inside the grating $(\xi>0)$. The two-color simulton, however, reduces its propagation speed with time (Fig. 7), tending to a stationary state. This result, rather surprising in terms of intuitive considerations on momentum conservation, can be explained on physical grounds by looking at the initial stages of the simulton formation, i.e. for $\tau<400$. A fraction of the generated SH freely propagates away from the input boundary $(\xi=0)$; this characterizes the low amplitude SH fields wherever generated by unbound $\omega$ components, i.e. outside the simulton. The corresponding unbound $\omega$ components, conversely, resonate with the Bragg grating and are subject to reflection within the DFBG, eventually counterpropagating towards the two-color 


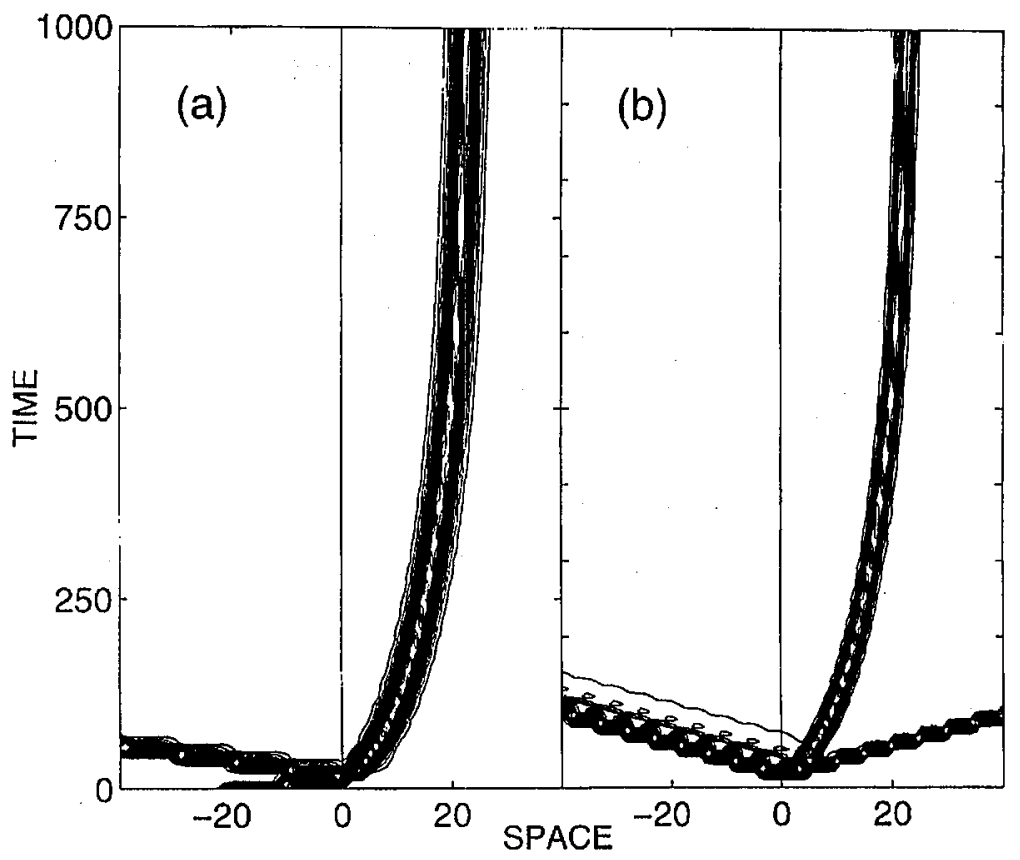

Fig. 6. Long range dynamics after the excitation of a slow gap simulton in a singly resonant semi-infinite grating, from Eqs. (1) with $v=0.5, \kappa=0, \Delta_{1}=-0.7$, and $\Delta_{2}=2$; (a) FF, (b) SH.

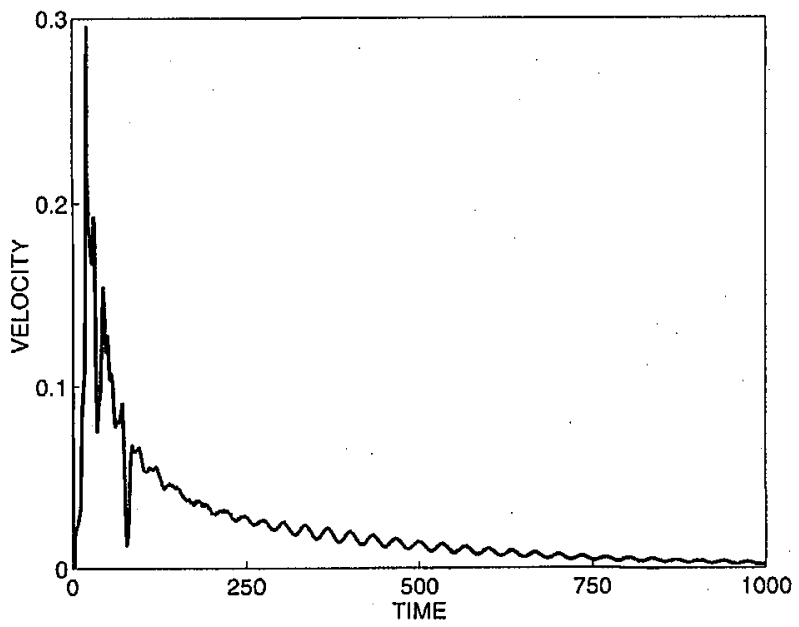

Fig. 7. Group velocity versus time for the "lazy" soliton graphed in Fig. 6.

gap-soliton. Both these contributions (locally adding or subtracting coherently) alter the overall momentum associated with the two-color gap-simulton, leading to the effect pictured in Figs. 6, 7. This small linear wave correction supports this 
physical picture only in the case of low velocity simultons such as those investigated here. This has led to the use of the term "lazy solitons" for such slowed-down localization states.

In conclusion, gap-solitons, encompassing two (or three, in general) frequency field components, can be excited in singly and doubly resonant photonic bandgap structures through multi-color energy localization. Such gap-simultons can be excited by launching a pulsed beam at the fundamental frequency into a Bragg grating, and evolve into slowly travelling eigenstates. Stationary states or "still" simultons can be obtained through inelastic collisions in drDFBGs, and can be exploited for all-optical memories capable of interrogation through the measurement of the time-of-flight of similar "bits". Stationary gap-simultons in srDFBGs can be the outcome of "lazy" solitons, which decelerate in propagation due to local interaction with linear waves.

\section{Acknowledgments}

This research was supported by the Italian Ministry for Scientific Research (1997 60\%).

\section{References}

[1] C.M. De Sterke, J.E. Sipe, in: Progress in Optics XXXIII, Ed. E. Wolf, Elsevier, Amsterdam 1994, Ch. III; Phys. Rev. A 39, 5163 (1989).

[2] M. Picciau, G. Leo, G. Assanto, J. Opt. Soc. Am. B 13, 661 (1996).

[3] C. Conti, S. Trillo, G. Assanto, Phys. Rev. Lett. 78, 2341 (1997).

[4] H. He, P.D. Drummond, Phys. Rev. Lett. 78, 4311 (1997).

[5] T. Peschel, U. Peschel, F. Lederer, B.A. Malomed, Phys. Rev. E 55, 4730 (1997).

[6] V.E. Zacharov, S.V. Manakov, Zh. Eksp. Theor. Fiz. Pis. Red. 18, 413 (1973) [Sov. JETP Lett. 18, 243 (1973)].

[7] Y.N. Karamzin, A.P. Sukhorukov, Pis'ma Zh. Eksp. Teor. Fiz. 20, 734 (1974) [JETP Lett. 20, 339 (1974)].

[8] C. Conti, S. Trillo, G. Assanto, Phys. Rev. E 57, R1251 (1998).

[9] C. Conti, G. Assanto, S. Trillo, Opt. Lett. 22, 1350 (1997).

[10] D. Baboiu, G.I. Stegeman, L. Torner, Opt. Lett. 20, 2282 (1995).

[11] C. Conti, S. Trillo, G. Assanto, Opt. Lett. 23, 334 (1998).

[12] C. Conti, G. Assanto, S. Trillo, Electron. Lett. 34, 689 (1998). 